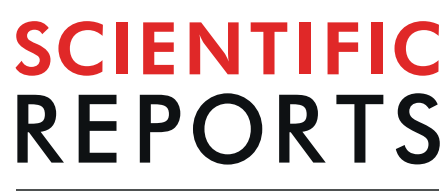

natureresearch

Received: 20 June 2019

Accepted: 24 August 2019

Published online: 11 September 2019

\section{An Experimental Approach to Rigorously Assess Paneth Cell $\alpha$-Defensin (Defa) mRNA Expression in C57BL/6 Mice}

Patricia A. Castillo ${ }^{1}$, Eric B. Nonnecke ${ }^{1}$, Daniel T. Ossorio ${ }^{1}{ }^{1}$, Michelle T. N. Tran ${ }^{1}$,

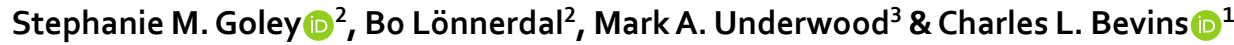

Abundant evidence from many laboratories supports the premise that $\alpha$-defensin peptides secreted from Paneth cells are key mediators of host-microbe interactions in the small intestine that contribute to host defense and homeostasis. $\alpha$-defensins are among the most highly expressed antimicrobial peptides at this mucosal surface in many mammals, including humans and mice; however, there is striking variation among species in the number and primary structure of $\alpha$-defensin paralogs. Studies of these biomolecules in vivo are further complicated by striking variations between laboratory mouse strains. Herein, we report an experimental approach to determine with precision and specificity expression levels of $\alpha$-defensin (Defa) mRNA in the small intestine of C57BL/6 mice through an optimized set of oligonucleotide primers for qRT-PCR assays and cloned CDNA plasmids corresponding to the Defa paralogs. This approach demonstrated marked differences in $\alpha$-defensin expression in C57BL/6 mice with respect to proximal/distal anatomical location and developmental stage, which have not been described previously. These data underscore the importance of careful attention to method (primer choice, proximal vs. distal location, and developmental stage) in analysis of antimicrobial peptide expression and their impact.

The intestinal mucosa of mammals is a site of remarkably complex and vitally important host-microbe interactions ${ }^{1}$. The intestinal tract harbors a diverse, abundant and dynamic group of microorganisms, which can have profound effects on host nutrition, physiology and immune response $\mathrm{e}^{2-9}$. While the host derives many benefits from mutualistic interactions with various gut microbes, disruption of this community, termed dysbiosis, creates niche opportunities for the proliferative expansion of noxious organisms that compromise homeostasis and can cause disease $\mathrm{e}^{9-23}$.

Accumulating evidence indicates that the host orchestrates mucosal homeostasis by accommodating and shaping the composition of contiguous microbial populations ${ }^{16,24-27}$. In the mammalian small intestine, one influential host factor of this ecosystem is a collection of proteins and peptides secreted by Paneth cells ${ }^{28-30}$. In humans, mice, and many other mammals Paneth cells are specialized epithelial cells located at the base of the crypts of Lieberkühn in the small intestine interdigitated between epithelial stem cells ${ }^{30}$. These secretory cells produce especially high quantities of antimicrobial peptides called $\alpha$-defensins ${ }^{29-31}$. In mice, $\alpha$-defensins were originally named "cryptdins" (for crypt defensins), which are orthologs of the human $\alpha$-defensins HD5 (DEFA5) and HD6 (DEFA6) ${ }^{28,32}$. Paneth cell $\alpha$-defensins are stored in cytoplasmic secretory granules together with other luminally delivered antimicrobials ${ }^{29}$. Secreted $\alpha$-defensins function to defend the intestinal mucosa from virulent microbes ${ }^{33,34}$, inhibit microbial translocation across the epithelium ${ }^{35}$ and help shape the composition of the colonizing microbiota ${ }^{24}$. Thus, Paneth cell $\alpha$-defensins serve critically important functions in homeostasis and host defense.

${ }^{1}$ Department of Microbiology \& Immunology, School of Medicine, University of California Davis, Davis, CA, 95616, USA. ${ }^{2}$ Department of Nutrition, College of Agricultural and Environmental Sciences, University of California Davis, Davis, CA, 95616, USA. ${ }^{3}$ Department of Pediatrics, School of Medicine, University of California Davis, Sacramento, CA, 95817, USA. Correspondence and requests for materials should be addressed to C.L.B. (email: clbevins@ ucdavis.edu) 
Mice are the most widely studied species to model acute and chronic disease ${ }^{36}$. Many valuable insights on enteric host-microbe interplay have stemmed from, and depend on, investigation of mouse models. Yet, while the in vivo dynamics of host-microbe interactions in the intestine can be profoundly influenced by Paneth cell $\alpha$-defensins, their characterization and biological analysis have been technically challenging due, in part, to their extensive repertoire within and across common laboratory mouse strains. Analysis of the $\alpha$-defensin gene locus in C57BL/6 mice identified a multitude of Defa genes, Defa-related genes and Defa pseudogenes that share very similar sequences ${ }^{37,38}$. The remarkable number of closely related $\alpha$-defensin gene paralogs in C57BL/6 mice renders analysis of expression of individual $\alpha$-defensin mRNA and protein difficult by routine quantitative reverse transcription-polymerase chain reaction (qRT-PCR) or antibody-based experimental approaches. Given the extensive reliance on $\mathrm{C} 57 \mathrm{BL} / 6$ mice for studies probing the influence of host genetics on host-microbe interactions, together with the importance of $\alpha$-defensins in the intestinal ecosystem, there remains a critical need for a robust and rigorous approach to analyze $\alpha$-defensin expression. To address this problem, Menendez, et al. ${ }^{39}$ made a significant initial advance for the field by identifying seven subgroups of expressed Defa genes in C57BL/6 mice. Isolation and structural characterization of the mature $\alpha$-defensin peptides corresponding to these subgroups had been previously reported by others ${ }^{38,40}$. This categorization enabled Menendez et al. to develop a set of PCR primer pairs that could amplify each of these Defa subgroups by qRT-PCR ${ }^{39}$.

Several years ago, our laboratory developed a robust qRT-PCR approach using external cDNA standards that readily permits quantitative comparisons of mRNA expression for genes encoding antimicrobial peptides in tissues from diverse specimens and experimental influences ${ }^{41}$. In this approach, we determine the absolute mRNA transcript numbers (based on external standard curves) as the primary data, not simply as a normalized ratio to a "housekeeping" gene (i.e., $\Delta \Delta \mathrm{CT})^{41}$. This approach is a bit more labor intensive, but addresses the recommendations by Bustin ${ }^{42}$, who demonstrated that normalization to "housekeeping" gene mRNA levels can compromise comparisons, or at times, lead to erroneous conclusions since expression levels of certain "housekeeping" genes (especially glyceraldehyde-3-phosphate dehydrogenase (Gapdh)) may vary by orders of magnitude among tissue sources, experimental conditions and/or tissue pathology $\mathrm{y}^{42,43}$.

With these issues in mind, the goals of the current study were to ( $i$ ) clone cDNA templates of $\alpha$-defensin encoding mRNA subgroups in C57BL/6 mice and develop a set of external standard quantitative curves for RT-PCR analysis, (ii) use these Defa cDNA templates to determine the specificity of each Defa PCR primer pair initially reported by Menendez, et al. ${ }^{39}$ and modify the primer sequences to substantially enhance specificity, and (iii) use the external standard curves to determine the absolute Defa mRNA abundance in C57BL/6 mice with respect to several experimental parameters. The latter included expression along the small intestinal tract, during postnatal development, in mice with genetic knockout of the gene encoding myeloid differentiation primary response 88 (MyD88), and in germ-free mice, as well as in mice following streptomycin treatment. We report the development of an optimized set of PCR primers and cloned cDNA standards, which together provide a set of qRT-PCR assays that are quantitative and specific for the paralog subgroups of $\alpha$-defensin genes in C57BL/6 mice (now expanded to eight). Using these assays, our data extend the previous reports of partial characterization of $\alpha$-defensin gene expression in this important mouse model. Importantly, our data reveal patterns of $\alpha$-defensin expression that vary dramatically in terms of anatomical location and developmental stage, which have not been previously elucidated. In addition, our results identify some key potential pitfalls that could arise from interpreting data from less rigorous analytical approaches.

\section{Results}

Sequence comparisons of Defa gene paralogs in C57BL/6 mice. The $\alpha$-defensin gene paralogs in C57BL/6 mice encode a collection of $\alpha$-defensin mRNA with remarkable sequence similarity (Fig. 1A,B and Supplementary Fig. S1) $)^{38,40}$. We refined the paralog grouping proposed by Menendez, et al. ${ }^{39}$ to include eight subgroups (Supplementary Table S1): Defa3 (Defa3 and Defa17), Defa5 (Defa5, Defa34, Defa35, Defa36 and Defa37), Defa20 (Defa20, Defa32, Defa33, and Defa2), Defa21 (single member), Defa22 (single member), Defa23 (Defa23, Defa27, and Defa31), Defa24 (Defa24 and Defa30) and Defa26 (Defa26 and Gm15292). We were able to establish discriminating assays for Defa21 and Defa22, yielding eight rather than the seven previously described subgroups $^{39}$. The individual members within each group had one or more discriminating nucleotides, but in the case of nonsynonymous differences, the encoded amino acid differences were subtle (Supplementary Fig. S1). Those amino acid substitutions were generally in the signal sequence or propeptide regions, except for Defa24 vs. Defa30 (which encode a conservative Leu/Met amino acid difference in the mature peptide), and Defa23 vs. Defa 27 (which encode conservative Ile/Val, Leu/Met, and Met/Ile amino acid differences in the mature peptide). Sequence similarity clustering (Clustal analysis) of the Defa mRNA sequences (Fig. 1B) and mature $\alpha$-defensin peptide sequences (Fig. 1C) show nearly identical patterns for these subgroups.

Development of new quantitative assays to assess C57BL/6 Defa mRNA expression with high specificity. In order to quantitate the expression of each of the eight C57BL/6 Defa paralog subgroups, we sought to determine absolute mRNA copy number using methodology previously published by our laboratory ${ }^{41}$. For this approach, we first cloned cDNA for the index member of each of the C57BL/6 Defa target paralog subgroups in order to develop standard curves for quantitative measure and rigorous assessment of assay specificity (see Methods). The serially diluted standard cDNAs for each Defa subgroup were then used as templates for qRT-PCR amplification using the gene-specific primers (Table 1). These PCR reactions were monitored to determine the threshold of product detection for each concentration of standard template and the data were used to create a standard curve to extrapolate values from experimental tissue samples. In order to ascertain if the Defa PCR primers (Table 1) were specific for their designated target, we tested each primer pair against the full panel of cDNA plasmid standards at a template concentration of $10^{5}$ copies per reaction (Fig. 2). Since each PCR primer 

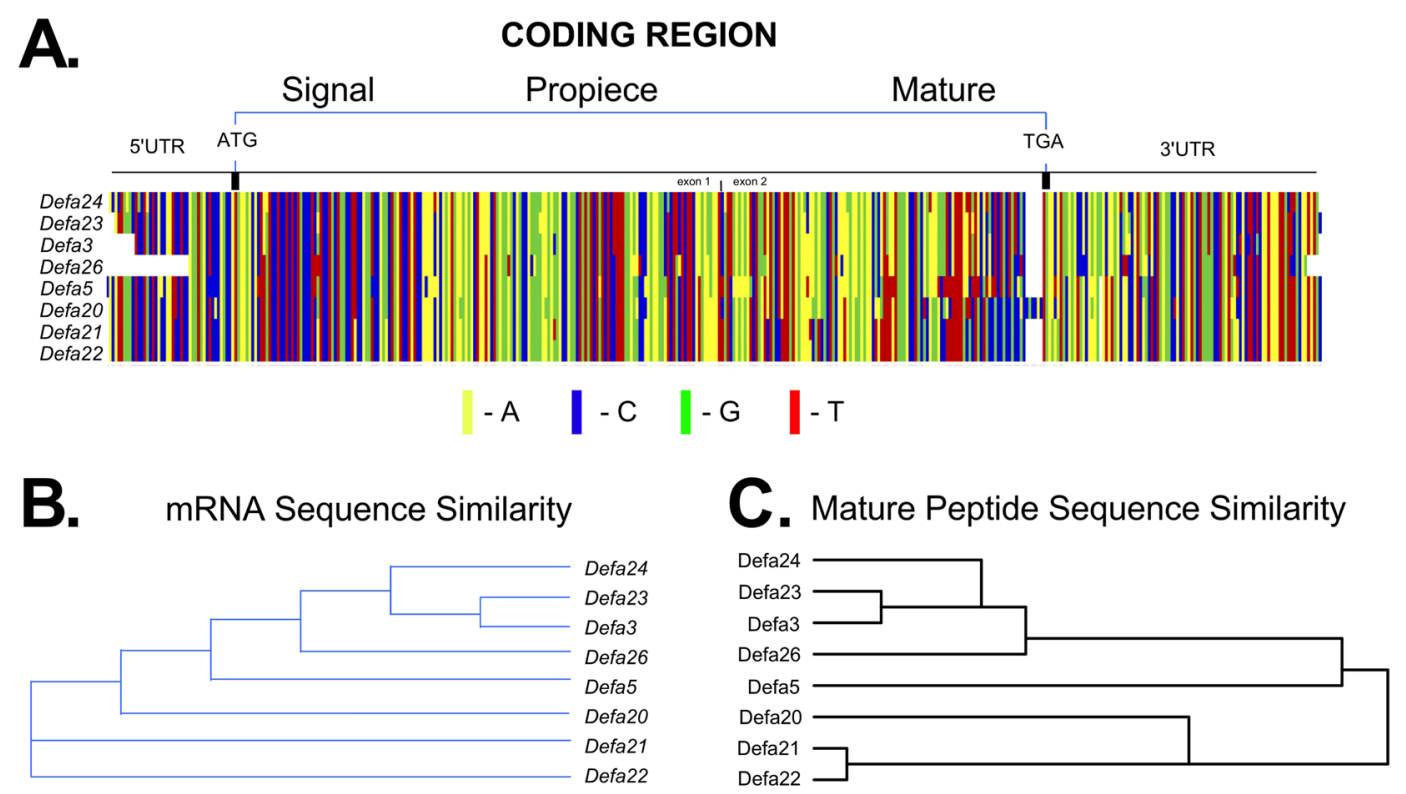

Figure 1. Sequence comparisons of C57BL/6 Defa mRNA and mature peptides. (A) Sequence alignment of eight Defa mRNA paralogs in C57BL/6 mice. The eight Defa mRNA paralogs are index members of the subgroups whose sequence alignment is presented in Supplementary Fig. S1. Accordingly the subgroups are: Defa3 (Defa3 and Defa17), Defa5 (Defa5, Defa34, Defa35, Defa36 and Defa37), Defa20 (Defa20, Defa32, Defa33, and Defa2), Defa21 (single member), Defa22 (single member), Defa23 (Defa23, Defa27, and Defa31), Defa24 (Defa24 and Defa30) and Defa26 (Defa26 and Gm15292). A high degree of sequence similarity uniformly distributed across coding and untranslated regions are evident. (B) Clustal similarity dendrogram of Defa mRNA sequences. NCBI accession numbers from sequences used in this analysis are listed in Supplementary Fig. S1. (C) Clustal similarity dendrogram of Defa mature peptide sequences. Sequences are from the literature ${ }^{38,40}$. Clustal analysis was performed by MacVector software using an unweighted pair group method with arithmetic mean.

\begin{tabular}{|l|l|l|l|l|}
\hline $\begin{array}{l}\text { Gene } \\
\text { Target }\end{array}$ & Primer Sequence & $\begin{array}{l}\text { Product } \\
\text { Length } \\
(\mathbf{B P})\end{array}$ & $\begin{array}{l}\text { Annealing } \\
\text { Temp } \\
\left({ }^{\circ} \mathbf{C}\right)\end{array}$ & $\begin{array}{l}\text { Reference } \\
\text { or } \\
\text { Source }\end{array}$ \\
\hline Defa3 & $\begin{array}{l}\text { F: ATCTGGTATGCTATTGTAGAAA } \\
\text { R: GTGGCCTCAGTACTCATGT }\end{array}$ & 147 & 62 & this work \\
\hline Defa5 & $\begin{array}{l}\text { F: TCAAAAAAGCTGATATGCTATTG } \\
\text { R: AGCTGCAGCAGAATACGAAAG }\end{array}$ & 106 & 58 & $\begin{array}{l}\text { F: ref }{ }^{39} \\
\text { R: this work }\end{array}$ \\
\hline Defa20 & $\begin{array}{l}\text { F: GAGAGATCTGATATGCTATTG } \\
\text { R: AGAACAAAAGTCGTCCTGAG }\end{array}$ & 86 & 62 & $\begin{array}{l}\text { F: this work } \\
\text { R: ref. } .\end{array}$ \\
\hline Defa21 & $\begin{array}{l}\text { F: GAGAGATCTGATCTGCCTTTG } \\
\text { R: CCTCTATTGCAGCGACGA }\end{array}$ & 45 & 64 & $\begin{array}{l}\text { F: ref } \\
\text { R: this work }\end{array}$ \\
\hline Defa22 & $\begin{array}{l}\text { F: AGCAGCCAGGGGAAGAG } \\
\text { R: CCTCTATTGCAGCGACGT }\end{array}$ & 124 & 64 & this work \\
\hline Defa23 & $\begin{array}{l}\text { F: TCTGGTATGCTATTGTAGAAC } \\
\text { R: GACAGCAGAGCGTGTATA }\end{array}$ & 95 & 62 & this work \\
\hline Defa24 & $\begin{array}{l}\text { F: GATCTGGTATGCTATTGTAGAG } \\
\text { R: GACAGCAGAGCATGTACAA }\end{array}$ & 97 & 64 & this work \\
\hline Defa26 & $\begin{array}{l}\text { F: ATTGTAGAAAAAGAGGCTGTAC } \\
\text { R: AGCAGAGTGTGTACATTAAATG }\end{array}$ & 81 & 62 & ref. ${ }^{39}$ \\
\hline
\end{tabular}

Table 1. Oligonucleotide primer sequences used for qRT-PCR analysis of $\alpha$-defensin (Defa) gene expression in C57BL/6 mice. PCR reaction conditions were $95^{\circ} \mathrm{C}$ for $5 \mathrm{~min}$, followed by 45 cycles of: denaturation at $95^{\circ} \mathrm{C}$ for $20 \mathrm{sec}$, annealing for $20 \mathrm{sec}$ at the temperature specified, and extension at $72^{\circ} \mathrm{C}$ for $30 \mathrm{sec}$. As indicated, the sequences were either from this study or Menendez, et al. ${ }^{39}$. F (forward/sense primer), R (reverse/antisense primer).

pair showed target specificity by amplifying only their intended cDNA target, we conclude that intended- vs. off-target amplification was $\geq 10^{5}$-fold in each case.

For analysis of most target genes, selection of PCR primers from adjacent exons allows easy discrimination of amplification from cDNA vs. (contaminating) genomic DNA templates, since genomic DNA will require less efficient amplification across an intron; however, the high sequence similarity of the Defa mRNA transcripts 
Defa3

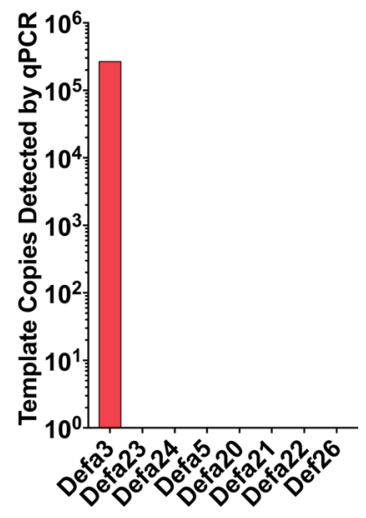

Defa22

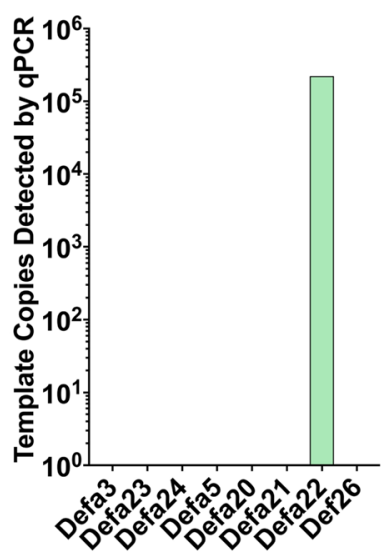

Defa5

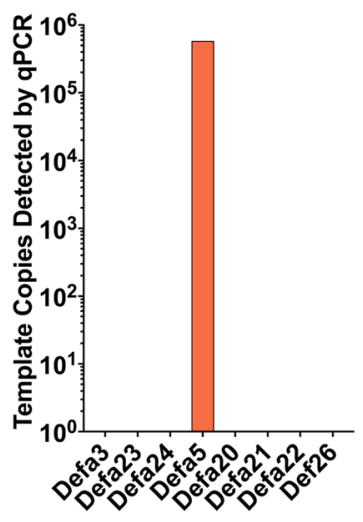

Defa23

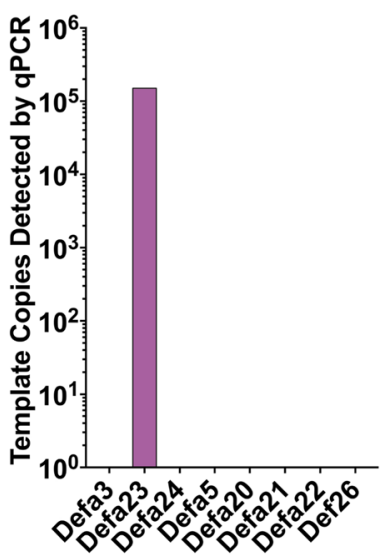

Defa20

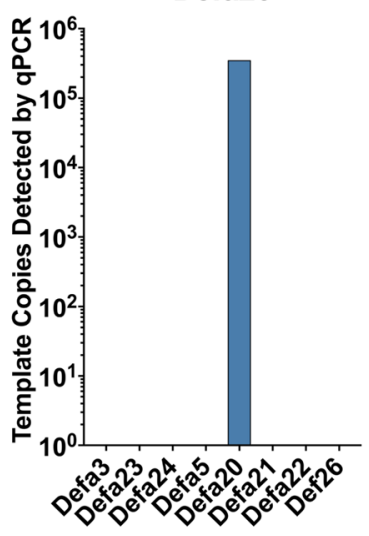

Defa24

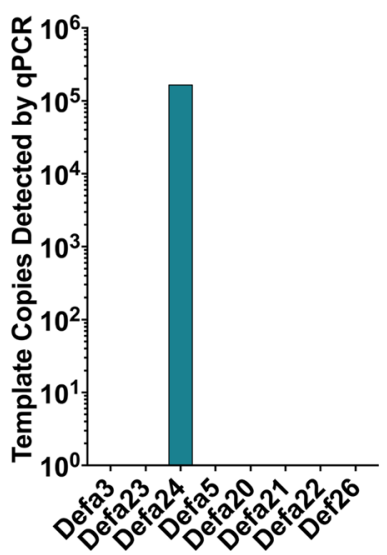

Defa21

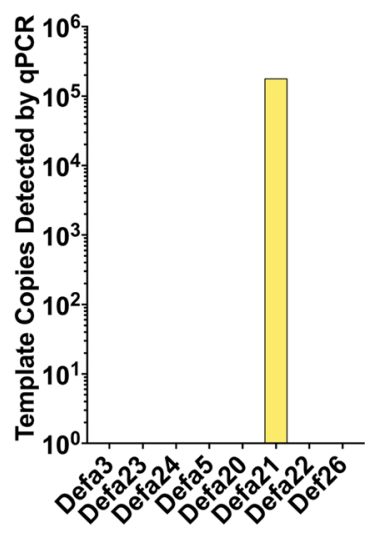

Defa26

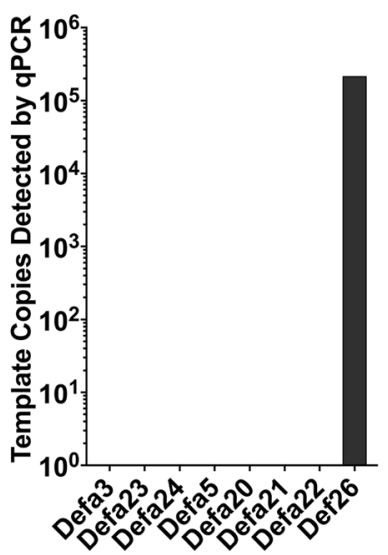

Figure 2. Analysis of specificity for the Defa qRT-PCR primer pairs. The eight pairs of Defa-targeting oligonucleotide primer pairs (Table 1) were used to amplify each of the eight plasmid cDNA standards present at $10^{5}$ copies per each reaction tube. Each panel of data represents reactions using the single pair of PCR primers indicated, and the $\mathrm{x}$-axis identifies the cloned cDNA templates used in the eight reactions. The plasmid cDNA standards were cloned, sequenced, and quantitated as described in the methods.

(Fig. 1) limited the selection possibilities for discriminatory gene-specific primers. Therefore, the PCR primer pairs for all except Defa22 were located within a single exon, giving rise to the possibility that trace contaminating genomic DNA could affect estimates of Defa mRNA expression at sites with low Defa gene expression (Supplementary Fig. S2). To examine this potential caveat, we treated isolated RNA specimens from colon, a tissue with negligible Defa gene expression, with DNase prior to cDNA synthesis. The apparent trace expression in the colon for Defa3, Defa 5 and Defa 21 ( $<10 \mathrm{e} 3 \mathrm{mRNA}$ copies per $10 \mathrm{ng}$ mRNA) was eliminated by DNase treatment (Supplementary Fig. S2). In parallel, estimates of beta-actin (Actb) expression, whose PCR primer pair spanned an intron (Table 2), did not change after DNase treatment of the colonic RNA supporting that the DNase treatment did not affect the quality of the RNA used in the analysis (Supplementary Fig. S2). Since Defa expression in the colon was either decreased or undetectable following DNase treatment, we conclude that the apparent low-level expression without DNase treatment is erroneous, and likely due to trace genomic DNA contamination of the isolated RNA specimens. By contrast, analysis of distal small intestine where Defa expression was abundant, showed DNase treatment had no detectable effect on estimates of Defa expression values (Supplementary Fig. S2). These control experiments highlight that caution is needed when interpreting and reporting low level mRNA expression ( $\leq 10 \mathrm{e} 3 \mathrm{mRNA}$ copies per $10 \mathrm{ng}$ mRNA) using most primers in Table 1. Our data indicate that either intron-spanning PCR primer pairs (Defa22) and/or DNase pretreatment of RNA is warranted to draw rigorous conclusions on low-level expression. Several additional control experiments for rigor and reproducibility of this qRT-PCR experimental approach are described in the Materials and Methods, and others were reported by our group previously ${ }^{41}$.

Anatomical expression of Defa mRNAs in the adult C57BL/6 mouse small intestine. Previous studies with FVB and BALB/c mice show regional variations for expression of some Paneth cell $\alpha$-defensin genes in the small intestine ${ }^{44-46}$. In particular, cryptdin $4^{44-46}$ and the cryptdin-related sequence $4 \mathrm{c}(\mathrm{Crs} 4 \mathrm{c} / \mathrm{Defcr} \text {-rs } 10)^{46}$ had higher expression in the distal small intestine (ileum) compared to more proximal sites. However, C57BL/6 mice do not express either cryptdin 4 or $\mathrm{Crs} 4 \mathrm{c}^{40,47}$ and regional analysis of expression for other $\alpha$-defensin genes is lacking. Therefore, we sought to determine quantitatively the anatomical expression of each Defa gene in adult 


\begin{tabular}{|l|l|l|l|l|}
\hline Gene & Primer Sequence & $\begin{array}{l}\text { Product } \\
\text { Length } \\
\text { (BP) }\end{array}$ & $\begin{array}{l}\text { Annealing } \\
\text { Temp }\end{array}$ & $\begin{array}{l}\text { Reference/ } \\
\text { Source }\end{array}$ \\
\hline Actb & $\begin{array}{l}\text { F: GCTGAGAGGGAAATCGTGCGTG } \\
\text { R: CCAGGGAGGAAGAGGATGCGG }\end{array}$ & 99 & 58 & ref. $^{77}$ \\
\hline$L y z 1$ & $\begin{array}{l}\text { F: GCCAAGGTCTACAATCGTTGTGAGTTG } \\
\text { R: CAGTCAGCCAGCTTGACACCACG }\end{array}$ & 86 & 58 & ref. $^{77}$ \\
\hline Lyz2 & $\begin{array}{l}\text { F: GGCTGGCTACTATGGAGTCAGCCTG } \\
\text { R: GCATTCACAGCTCTTGGGGTTTTG }\end{array}$ & 177 & 58 & ref. $^{46}$ \\
\hline Nod2 & $\begin{array}{l}\text { F: CGACATCTCCCACAGAGTTGTAATCC } \\
\text { R: GGCACCTGAAGTTGACATTTTGC }\end{array}$ & 123 & 58 & ref. $^{77}$ \\
\hline Mmp7 & $\begin{array}{l}\text { F: TTCAAGAGGGTTAGTTGGGGGACTG } \\
\text { R: TTGTCAAAGTGAGCATCTCCGCC }\end{array}$ & 152 & 58 & ref. $^{46}$ \\
\hline Slc10a2 & $\begin{array}{l}\text { F: TTGCCTCTTCGTCTACACC } \\
\text { R: CCAAAGGAAACAGGAATAACAAG }\end{array}$ & 107 & 58 & this work $^{4}$ \\
\hline
\end{tabular}

Table 2. Oligonucleotide primer sequences used for qRT-PCR analysis of gene expression in C57BL/6 mice. The primer target sequences were in exons so as to span an intron in the genomic sequence. PCR reaction conditions were $95^{\circ} \mathrm{C}$ for $5 \mathrm{~min}$, followed by 45 cycles of: denaturation at $95^{\circ} \mathrm{C}$ for $20 \mathrm{sec}$, annealing for $20 \mathrm{sec}$ at $58^{\circ} \mathrm{C}$, and extension at $72^{\circ} \mathrm{C}$ for $30 \mathrm{sec} . \mathrm{F}$ (forward/sense primer), $\mathrm{R}$ (reverse/antisense primer).

C57BL/6 mouse small intestine by analyzing adjacent $3 \mathrm{~cm}$ sections along the proximal to distal axis, giving a total of ten sections (Fig. 3A). Absolute quantities of each Defa mRNA were determined by the qRT-PCR approach outlined above. The data from this analysis (Fig. 3B,D) yielded several interesting findings. Defa3, Defa5, Defa23, Defa 24 and Defa26 expression were relatively uniform along the tract (Fig. 3D). Among these, Defa24 showed remarkably high expression $\left(5 \times 10^{6}-1 \times 10^{7} \mathrm{mRNA}\right.$ copies per $\left.10 \mathrm{ng} \mathrm{mRNA}\right)$ in all sections throughout the small intestine (Fig. 3D,E). Defa3, Defa5 and Defa23 were also abundant, but about 5-fold less than Defa24 (Fig. 3B,D). In contrast to the uniform expression of those Defa genes, Defa20, Defa21 and Defa22 mRNA expression showed marked regional differences, with dramatically higher expression (approximately 100-fold) in the distal sections (Fig. 3B). Defa26 was found to be the least abundant among the $\alpha$-defensin mRNA at all locations (Fig. 3D,E). The patterns of expression for Defa mRNA parallel the sequence similarity clustering (Clustal analysis) of mRNA (Fig. 1B) and mature $\alpha$-defensin peptide sequences (Fig. 1C) highlighting sequence associated patterns of expression.

Expression of other host-microbe related genes in the small intestine were also analyzed in the ten sections, including regenerating islet-derived 3 gamma (Reg3g), the two lysozyme paralogs ( $L y z 1$ ("Paneth cell") and $L y z 2$ ("macrophage"), matrix metalloproteinase-7 (Mmp7, the $\alpha$-defensin processing enzyme in mice ${ }^{33,34}$ ), and the nucleotide-binding oligomerization domain containing protein 2 (Nod2, which recognizes intracellular peptidoglycan), as well as control genes Actb and the apical sodium-dependent bile acid transporter (Slc10a2). All of these genes, except Slc10a2 were expressed at a relatively constant level along the small intestine (Fig. 3F). Previous studies by others established that Slc10a2 is expressed much more prominently in the distal small intestine, compared to proximal sites ${ }^{48}$. The qPCR expression data for Slc10a2 obtained here (Fig. 3F) reflected that pattern and served as a control to verify the proximal/distal orientation of the intestinal sections.

Defa expression levels were very similar across the first 3-4 proximal sections; likewise, the most distal 3-4 sections showed similar levels of Defa expression (Fig. 3B,D). To simplify subsequent analysis of anatomic expression, we decided to analyze $10 \mathrm{~cm}$ of the most proximal and $10 \mathrm{~cm}$ of the most distal segments of small intestine. Consistent with data in Fig. 3, Defa3, Defa23, Defa24 and Defa26 were expressed at similar levels in the proximal and distal small intestine (Fig. 4A), while the differentially expressed Defa mRNA were more abundant in the distal region (Fig. 4B). Thus, Defa5 mRNA is 10-fold more abundant, whereas Defa20, Defa21 and Defa22 are 100 -fold more abundant in distal sections. In the proximal small intestine, Defa mRNA expression was dominated by Defa3, Defa5, Defa23, Defa24, whereas in the distal region the relative Defa mRNA expression was more proportionately distributed (Fig. 5A). The sum (total) number of Defa transcripts in the distal intestine was $\sim 5$-fold higher than in the proximal small intestine (Fig. $5 \mathrm{~F}$ ). No significant differences were detected when comparing data from male and female mice (data not shown).

Defa expression in germ-free and antibiotic treated mice. As compared to conventional mice, mice reared under germ-free conditions have profound alterations in both their innate and adaptive immune systems ${ }^{49-52}$. For example, the expression of Reg $3 g$ in the small intestinal epithelium of ex-germ-free mice is markedly induced ( 10 - to 100 -fold) upon colonization with a conventional microbiota through a MyD88-dependent mechanism ${ }^{35,46,53}$. Previous studies have indicated that expression of Paneth cell $\alpha$-defensins has less dependence ( $\sim$ - to 10 -fold) on the presence of a conventional microbiota ${ }^{40,45,46,53,54}$. Using our quantitative approach, the absolute copy number of each Defa mRNA was determined in $10 \mathrm{~cm}$ sections of the proximal and distal small intestine of adult C57BL/6 mice (Fig. 5). When examining the total abundance of Defa mRNA there were significantly fewer Defa transcripts ( 25-fold) in the small intestine of germ-free mice at both the proximal and distal sites compared to conventionally housed controls (Fig. 5E,F). The relative pattern of abundance of individual Defa mRNA in the proximal and distal small intestine appeared similar to that of untreated control mice (Fig. 5A,B). By weighted PCoA, Defa expression in germ-free mice clustered away from controls, owing to differences in absolute expression levels (Fig. 5I,J). We conclude that Defa mRNA levels are significantly lower in both the proximal and distal small intestine of germ-free mice, but that the relative pattern of Defa expression is similar. In control experiments, expression of Reg $3 g$ in the conventional mice was at levels comparable to Actb, whereas 

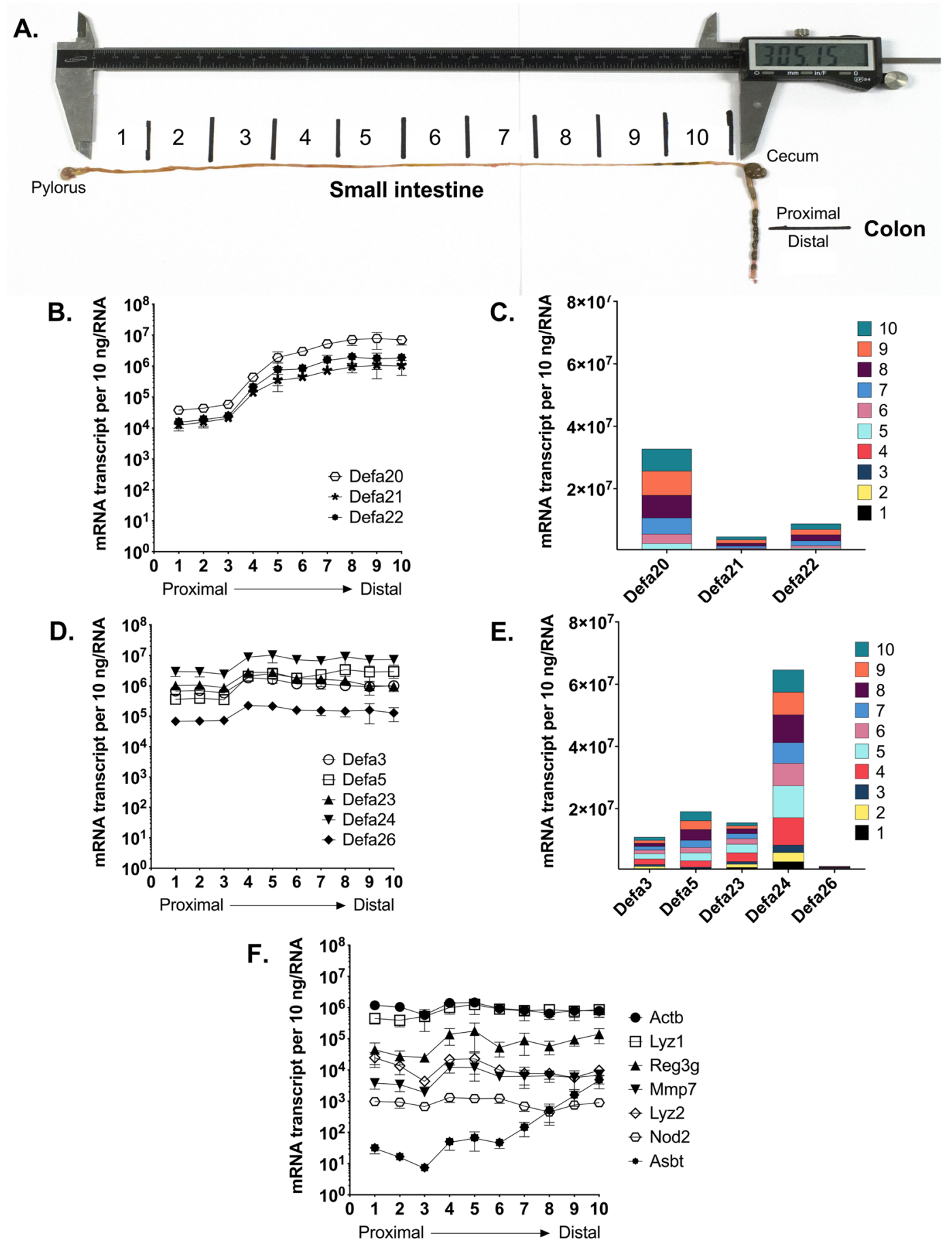

Figure 3. Absolute quantification of Defa mRNA in adjacent sections along the small intestinal tract of wild type C57BL/6 mice. (A) Image depicts the most proximal to the most distal sections $(3 \mathrm{~cm}$ each) of small intestine procured for analysis. (B,D) Quantitative RT-PCR analysis and (C,E) relative percent of each mouse Defa mRNA gene analyzed in tissue in $3 \mathrm{~cm}$ sections from the most proximal to most distal small intestine. (F) Quantitative RT-PCR analysis of other Paneth cell products and control genes in $3 \mathrm{~cm}$ sections from the most proximal to the most distal small intestine. PCR oligonucleotide primers were described in Table 1 (for panels $\mathrm{B}, \mathrm{D}$ ) and Table 2 (for panel F). The error bars represent standard error of the mean, $\mathrm{N}=4$ mice.

in the germ-free animals Reg3g expression was lower by approximately 10- to 100 fold (Supplementary Fig. S3), consistent with previous reports ${ }^{46,53,55,56}$.

Since mice are routinely orally gavaged with streptomycin before oral challenges with pathogens such as $S$. Typhimurium to overcome microbiota-mediated resistance to infection ${ }^{57-60}$, we sought to determine if streptomycin treatment modulated Defa mRNA expression. Adult C57BL/6 mice were orally gavaged with streptomycin $(1 \mathrm{mg} / \mathrm{g}$ body weight), which is a typical protocol prior to inoculation with enteropathogens. After four days, the 
A.

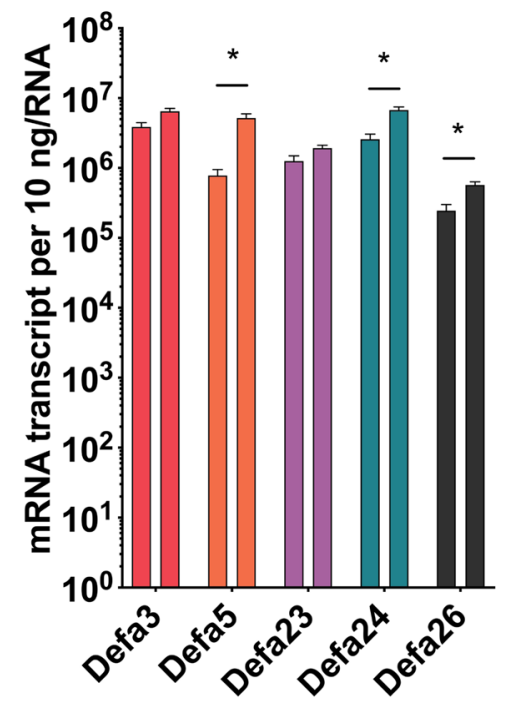

B.

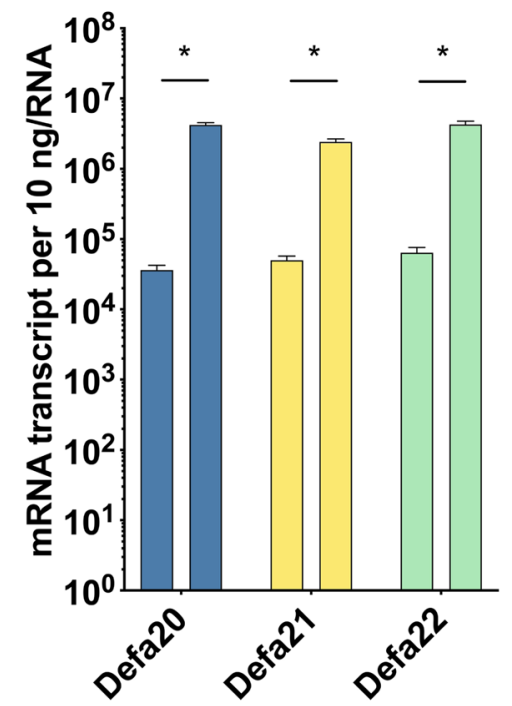

Figure 4. Quantitative comparison of Defa mRNA expression in proximal and distal small intestine of wildtype C57BL/6 mice. Quantitative real-time PCR analysis showing absolute quantification of each mouse Defa mRNA analyzed in tissue from the most proximal (left bars) and distal $10 \mathrm{~cm}$ sections (right bars) of the small intestine of wild type C57BL/6 mice. (A) Expression of Defa3, Defa5, Defa23, Defa24 and Defa 26 paralog groups which have less pronounced anatomic expression differential. (B) Expression of Defa20, Defa21 and Defa 22 paralog groups which have greater anatomic expression differential. The error bars represent standard error of the mean. A Mann-Whitney test was used for non-parametric statistical analysis ( $p$ values less than 0.05 were considered significant, $\mathrm{N}=4$ mice).

absolute copy number of each Defa mRNA was determined in $10 \mathrm{~cm}$ sections of the proximal and distal small intestine. No statistically significant differences in Defa mRNA expression were detected in streptomycin-treated mice compared to untreated control mice in terms of total abundance of Defa mRNA in either the proximal or distal small intestine (Fig. 5G). The relative abundance pattern of the Defa mRNA (Fig. 5C) was similar and on weighted PCoA plots, the data points for the specimens from streptomycin-treated mice cluster with untreated controls in both the proximal and distal small intestine (Fig. 5I,J). Thus, while Defa mRNA expression varies when comparing germ-free to conventional mice, expression patterns are resilient to less profound transient perturbations in luminal microbes.

Defa expression in MyD88 knockout mice. MyD88 is an adaptor protein required for signaling from the TLR/IL-1 family of receptors. The expression of Reg $3 g$ in the small intestine is critically dependent on this biomolecule $35,53,61$. A previous study reported that MyD88 deficient mice had significantly lower expression of all Defa genes (3- to 20 -fold) in the distal small intestine ${ }^{39}$, whereas others reported no significant differences in small intestinal Defa gene expression in $M y D 88$ deficient compared to controls ${ }^{61}$. In the present study, absolute copy number of each Defa mRNA was determined in both the proximal and distal small intestine of $M y D 88$ knockout mice (Fig. 5D,H). No statistically significant differences in Defa mRNA expression were detected in MyD88 knockout mice compared to control C57BL/6 mice. Thus, the total abundance of Defa mRNA in the proximal and distal small intestine was indistinguishable from control mice (Fig. $5 \mathrm{H})$, the relative abundance pattern for the Defa mRNA transcripts (Fig. 5D) was similar, and data points from the MyD88 knockout cluster with data points from control wildtype mice on weighted PCoA plots (Fig. 5I,J). Consistent with previous reports ${ }^{35,55,61,62}$, expression of Reg3g in both the proximal and distal small intestine was approximately 10- to 100-fold lower in MyD88 knockout mice compared to wildtype controls (Supplementary Fig. S3).

Dynamics of Defa gene expression during postnatal development of C57BL/6 mice. Previous studies using outbred Swiss mice, and inbred FVB and BALB/c mice have shown that Defa genes can be detected in neonatal small intestinal tissue and that their expression increases until adulthood ${ }^{45,63-65}$. To address how small intestinal expression of the Defa genes change during development using our qRT-PCR approach, we isolated samples of proximal and distal small intestine from pre-weanling (postnatal days 5, 10, and 20) and adult (postnatal day 50) mice. In both proximal and distal small intestine, collective transcript levels of Defa genes increased from postnatal days 5 to 50 reaching levels of $10^{5}-10^{7}$ copies per 10 ng RNA in proximal and distal small intestine, respectively (Fig. 6A). The increased abundance was most marked from days 20 to 50 for all Defa genes. Increased expression was also observed when comparing days 5 to 10 for all Defa genes with the exception of Defa24 and Defa3. More modest changes for all Defa genes were observed between postnatal days 10 and 20. Similar to the Defa genes, Reg3g, and Lyz1 (Paneth cell lysozyme) exhibited increased expression from postnatal day 5 to 50, with Reg3g showing high expression that plateaued by postnatal day 20 (Fig. 6B). However, the magnitude of change was lower for both Reg3g and Lyz1 ( 10-fold) compared to the Defa genes ( 100-fold), in both proximal and distal small intestine. Little change in Reg3g and $L y z 1$ expression was detected in the colon during 
A.
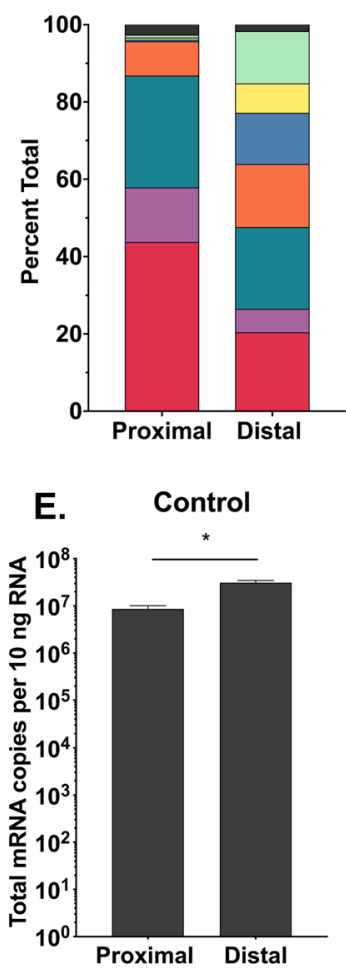

B.

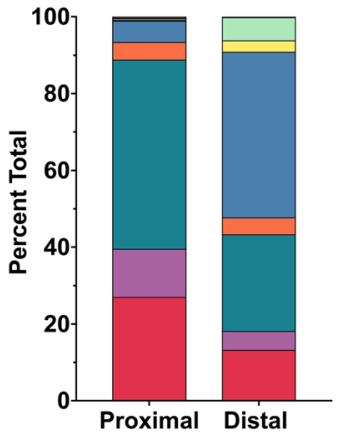

F. Germ Free

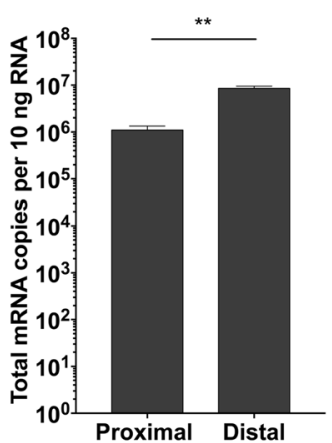

C. Streptomycin

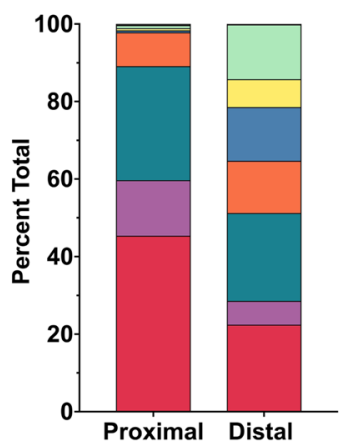

G. Streptomycin

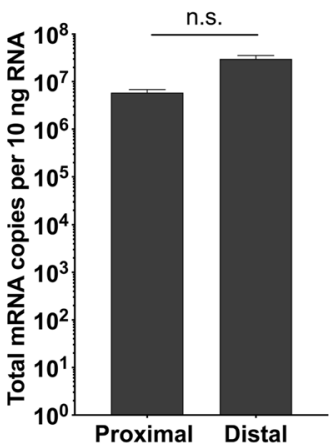

D. MyD88

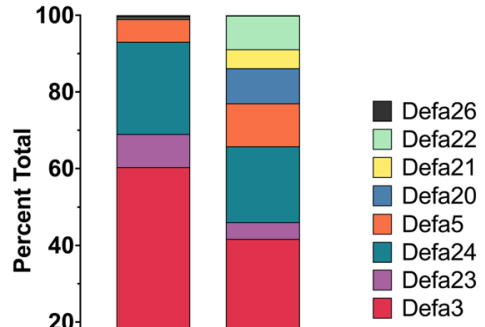

I.

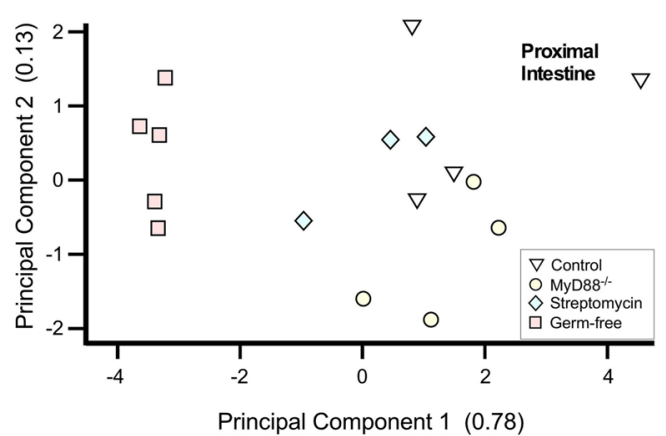

J.

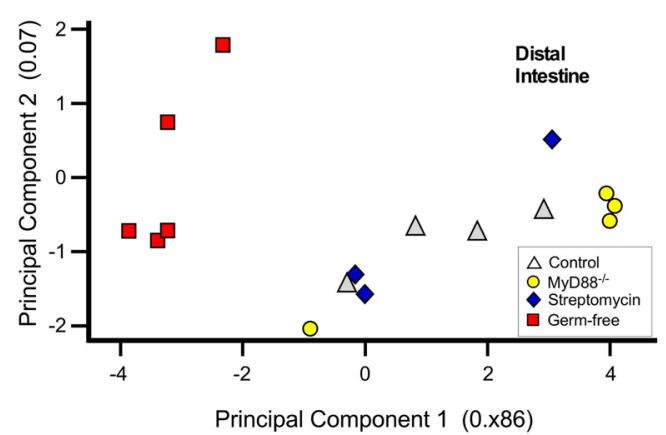

Figure 5. Expression of Defa mRNAs in the small intestine C57BL/6 mice. Quantitative real-time PCR analysis showing relative percent of each mouse Defa mRNA expressed (A-D) and an arithmetic sum of all Defa transcripts detected $(\mathbf{E}-\mathbf{H})$ in the most proximal and distal $10 \mathrm{~cm}$ tissue sections of the small intestine of $(\mathbf{A}, \mathbf{E})$ wild type control, $(\mathbf{B}, \mathbf{F})$ germ-free, $(\mathbf{C}, \mathbf{G})$ streptomycin treated and $(\mathbf{D}, \mathbf{H}) \mathrm{My} D 88$ gene knockout mice. Weighted principle component analysis of data from A-D for the most proximal (I) and distal (J) $10 \mathrm{~cm}$ sections of the small intestine. For E-H, the error bars represent standard error of the mean. A Mann-Whitney test was used for non-parametric statistical analysis ( $\mathrm{p}$ values less than 0.05 were considered significant, $\mathrm{N}=4$ for untreated and MyD88, $\mathrm{N}=3$ for streptomycin, and $\mathrm{N}=5$ for germ-free). ${ }^{*} \mathrm{p}<0.05, * * \mathrm{p}<0.01$.

development (Supplementary Fig. S4). The expression of Actb mRNA was relatively consistent at all anatomic sites and independent of age.

\section{Discussion}

Paneth cell $\alpha$-defensins mediate critically important functions for the intestinal ecosystem, including helping to shape the composition of the colonizing microbiota, as well as protecting the host from food and water-borne pathogen ${ }^{28-30}$. Determining expression levels of $\alpha$-defensins can be experimentally challenging, in part, because the significant variation in sequence between mammalian species requires species-specific reagents. Special challenges are encountered with investigations of mice, the most commonly utilized laboratory model for probing host-microbe interaction. Owing to extensive gene duplication in the defensin gene locus, common laboratory mouse strains harbor exceptionally variable collections of $\alpha$-defensin gene paralogs, often with high sequence similarity $37,38,40,66$. Together these features make the design of discriminating assays difficult. Our approach uses a collection of cloned external standards that permit not only quantitative measure, but also provide a means 

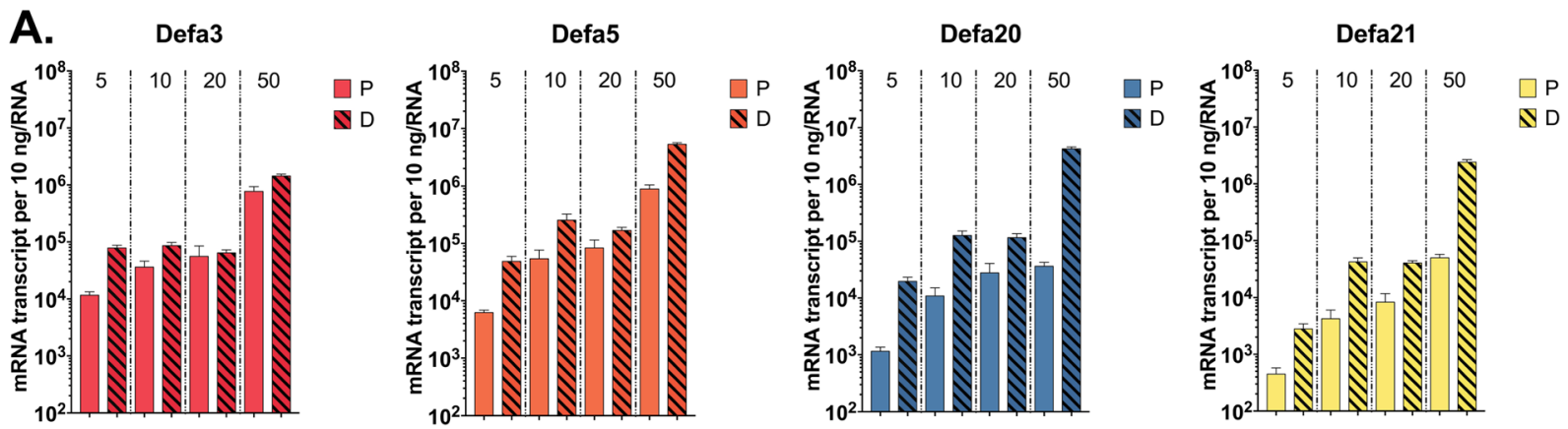

Defa22

Defa23
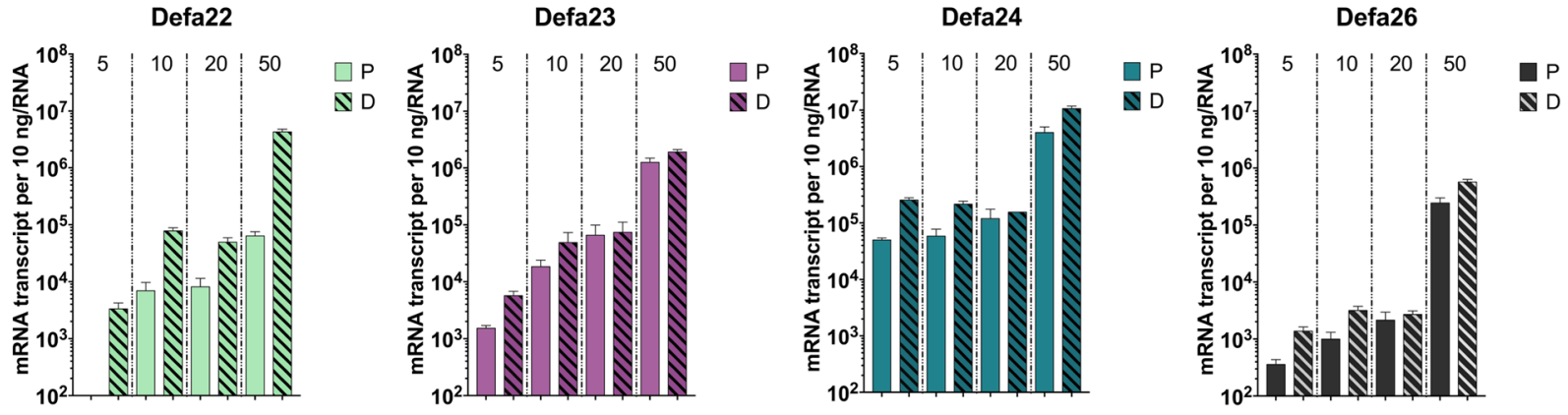

B.

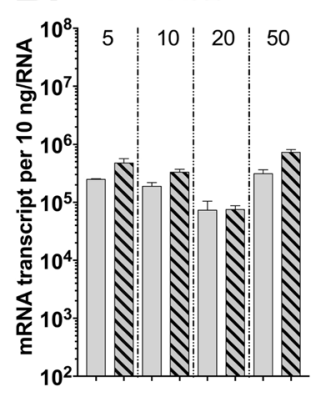

Reg3g

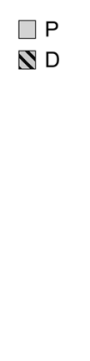

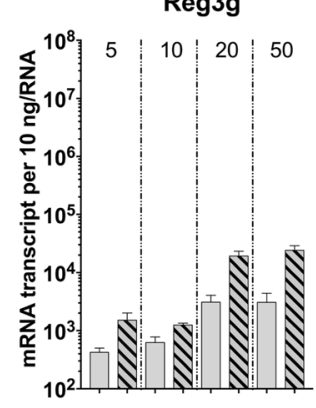
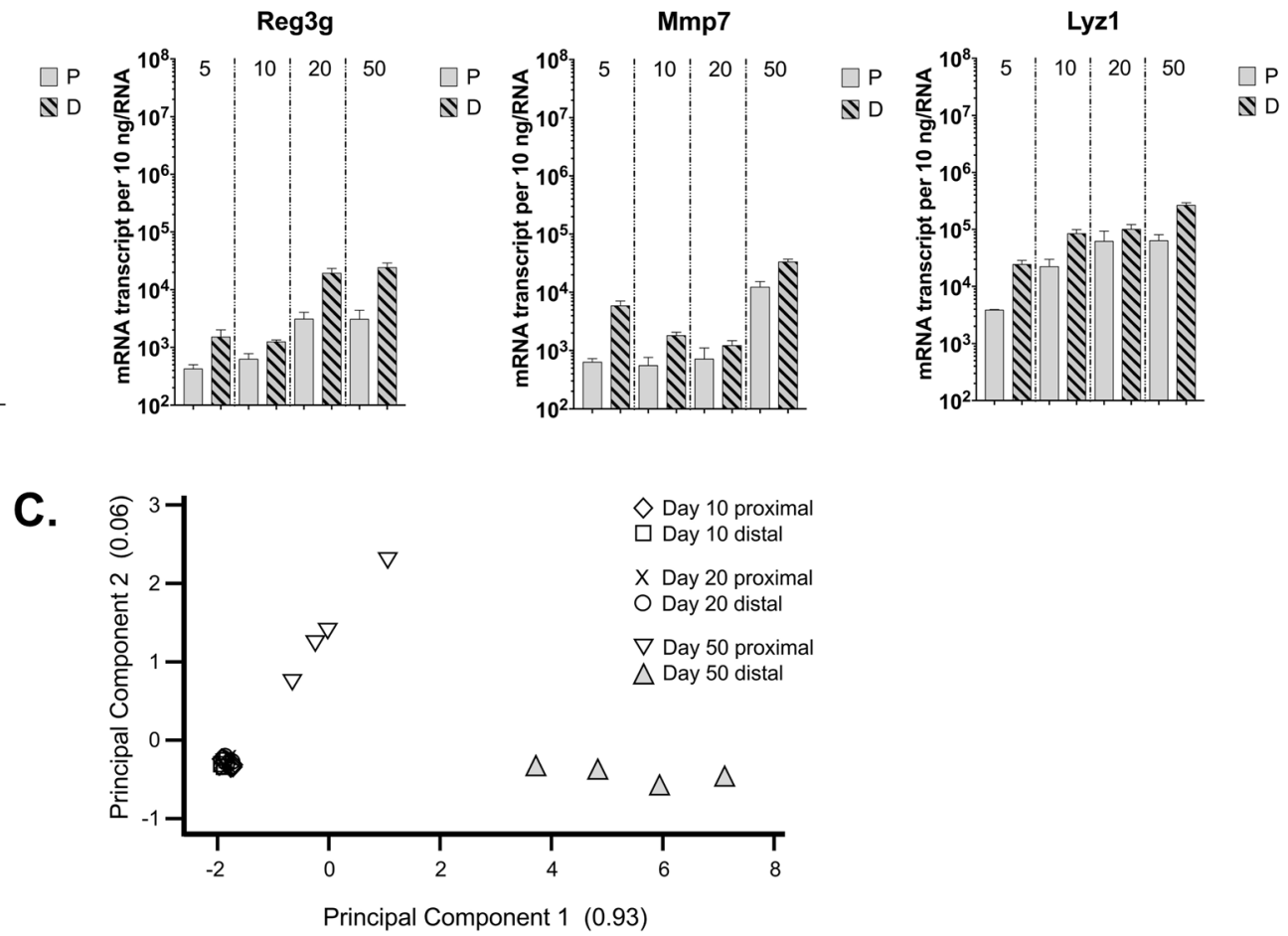

Figure 6. Expression of Defa mRNA in the small intestine during development of C57BL/6 mice. (A) Quantitative RT-PCR analysis of Defa mRNA and (B) other Paneth cell products and control genes analyzed in the most proximal $10 \mathrm{~cm}$ to the most distal $10 \mathrm{~cm}$ sections of the small intestine in post-natal day $5,10,20$ and 50. Error bars represent standard error of the mean, $N=4$ mice. (C) Weighted principal component analysis of Defa mRNA levels in the most proximal and distal $10 \mathrm{~cm}$ of the small intestine on postnatal day 10, 20, 50 .

to directly assess assay specificity. The PCR primer pairs developed here (Table 1) for the qRT-PCR assays were initially based on those reported by Menendez ${ }^{39}$. Our panel of cDNA clones for each of the eight target Defa subgroups enabled meticulous optimization of the primer pairs used in this study, which provided target specificity of $\geq 10^{5}$-fold compared to off-target PCR amplification (Fig. 2).

One of the important findings reported here is the anatomic distribution of expression along the length of the small intestine (Fig. 3), yielding several notable expression patterns: (i) The total abundance of Defa mRNA was lower in the most proximal sections; (ii) Defa24 and Defa20 were the most abundant mRNA in any of sections; 
(iii) Expression of Defa20, Defa21 and Defa22 mRNA was dramatically ( 100-fold) increased in the more distal segments. These regional expression differences are consistent with data from previous studies in outbred Swiss mice ${ }^{44}$, and inbred $129 / \mathrm{Sv}^{65}, \mathrm{BALB} / \mathrm{c}^{45}$ and FVB mice ${ }^{46}$, as well as a recent study using single-cell RNA sequence analysis of small intestine in C57BL/6 mice $^{67}$. Higher Defa mRNA expression in the distal small intestine could not be attributed solely to numbers of Paneth cells per crypt, which are higher distally by only about 3-fold ${ }^{63,68}$.

An important practical point regarding experimental reproducibility and rigor should be emphasized. Because of the substantial variation in expression of Defa genes along the small intestinal tract, careful sample procurement is critically important in experiments aimed to assess Defa mRNA abundance between treatment groups. For example, estimates of Defa20, Defa21 and Defa 22 mRNA levels could erroneously appear to vary significantly as a result of experimental manipulation, while measured differences could be, in fact, dependent solely on imprecise anatomic sampling since expression levels vary in proximal vs. distal sections $\sim 100$ - to 1000 -fold.

Whereas this investigation focused on small intestinal $\alpha$-defensin gene expression assessed at the mRNA level, the expression data reported here are supported by previous investigations from two laboratories reporting the isolation and characterization of the small intestinal $\alpha$-defensin peptides from C57BL/6 mice ${ }^{38,40}$, including studies of in vitro antimicrobial activity ${ }^{38}$. Of note, the $\alpha$-defensins showing the striking pattern of much higher mRNA expression in the distal small intestine, Defa20, Defa21, and Defa22 (Figs 3 and 4), encode mature peptides with distinguishable features in primary structure (Fig. 1 and Supplementary Fig. S5). These $\alpha$-defensins have extended C-termini containing multiple cationic amino acid residues and a reduction in the distance between disulfide-participating Cys(IV) and Cys(V) by three amino acid residues (Supplementary Fig. S5) ${ }^{38,40}$. The overall sequence of mature Defa20, Defa21, and Defa22 peptides are more similar with one another, and differ in sequence from the other five mature $\alpha$-defensins (Fig. 1 and Supplementary Table S2). These latter five (Defa3, Defa5, Defa23, Defa24 and Defa 26) are more similar with one another than the others and more uniformly expressed. It is likely that the marked differences in expression of the structurally dissimilar $\alpha$-defensins along the small intestine have implications on their protective and/or homeostatic functions. Genomic sequence analysis using ClustalW nucleotide alignments and Pustell matrix blots could not readily discriminate between these two groups (Supplementary Fig. S6), leaving the mechanism for differential gene expression an open question.

Another notable change in Defa gene expression reported here is the pattern during postnatal development, with most notable increases detected for all eight subgroups of Defa genes when transitioning from postnatal day 20 (weaning period) to adult mice (Fig. 6). The transcript levels of Defa genes increased $~ 10$ - to 100-fold during this developmental window, whereas Reg3g, Lyz1 (Paneth cell lysozyme), and Mmp7 expression levels increased modestly ( 5- to 10-fold) during this developmental window. The numbers of Paneth cells per crypt in this developmental window of C57BL/6 mice increase from 3-4 per crypt in the days prior to weaning to 5-6 per crypt in the weeks after weaning ${ }^{69,70}$, so much of the observed increase in mRNA levels is attributable to increased gene expression. These changes in gene expression during development in C57BL/6 mice are in-line with previous studies using outbred Swiss, and inbred FVB and BALB/c mice ${ }^{45,46,63-65}$. The high incidence of bacterial diarrhea as a cause of mortality in newborns and young children is likely due to developmental immaturity of the innate immune system, possibly including $\alpha$-defensins.

Compared to conventionally reared control mice, the collective total abundance of Defa transcripts was lower ( 25 -fold) in the small intestine of germ-free mice at both the proximal and distal sites (Fig. 5). The numbers of Paneth cells per crypt in germ-free mice is modestly lower than in controls ( 4.1 vs. 6.4 per crypt, respectively) $)^{69}$, so much of the observed difference in mRNA levels is attributable to gene expression. These data are in line with previous studies probing the effects of a germ-free environment on quantitative expression of Paneth cell $\alpha$-defensins in BALB/c, NMR/KI, 129/Sv and outbred Swiss mice ${ }^{40,45,46,54}$. Whereas chronic exposure to antibiotics affects Defa expression ${ }^{39}$, the more subtle and transient perturbations in microbiota associated with the commonly employed oral gavage with streptomycin ${ }^{71}$ in conventionally reared control mice resulted in no perceptible change in $\alpha$-defensin expression (Fig. 5), although transient subtle alterations at other timepoints cannot be ruled out. In a previous study of C57BL/6 mice with more prolonged antibiotic exposure (streptomycin $(450 \mathrm{mg} / \mathrm{l}) \mathrm{in}$ drinking water for four days), decreased expression of Defa5 (2-fold), Defa20 (30-fold) and Defa23 (4-fold) in the distal small intestine were reported ${ }^{39}$.

We also report data addressing a controversial issue in the literature on Defa expression in MyD88 deficient C57BL/6 mice. Our determination of absolute copy number for each Defa mRNA in both the proximal and distal small intestine of $M y D 88$ knockout mice compared to wildtype controls found no significant differences (Fig. 5). As a control, our analysis of expression of Reg $3 g$ in both the proximal and distal small intestine showed approximately 10- to 100-fold lower mRNA levels in MyD88 knockout mice compared to controls (Supplementary Fig. S3), consistent with several published reports ${ }^{35,55,61,62}$. Thus, our data on Defa expression in MyD88 deficient C57BL/6 mice are in line with the results of Stockinger, et al. ${ }^{61}$, rather than an earlier report of significantly lower Defa gene expression in the distal small intestine of $M y D 88$ deficient mice ${ }^{39}$.

qRT-PCR is a widely utilized method of determining mRNA expression levels and the approach reported here should aid in investigations of host-microbe dynamics in C57BL/6 mice. We concur with the recommendation of Bustin $^{42}$ that housekeeping gene expression levels should be reported relative to total tissue RNA, rather than as a normalizing denominator (i.e., $\Delta \Delta \mathrm{CT}$ ). When values for target gene mRNA levels are normalized to a housekeeping gene information is lost, comparisons between experiments are compromised and erroneous results can stem from differences in housekeeping mRNA levels that might vary among different tissues or with disease status $^{42,43}$. However, even if the oligonucleotide PCR primers of Table 1 are used in qRT-PCR assays relying on control gene expression (i.e., $\Delta \Delta \mathrm{CT}$ ) rather than the more elaborate external standard comparators used here, specificity of target Defa gene assessment is still possible in C57BL/6 mice. A caveat must be noted, however. Because of the extensive nucleotide similarity within the Defa mRNA of C57BL/6 mice (Fig. 1), the discriminating PCR primer pairs that were selected and optimized for this study (Table 1) were contained within a single exon, except for the intron-spanning pair targeting Defa22. As highlighted in Supplementary Fig S2, when using 
the primer pairs that do not span an intron, DNase treatment of RNA prior to cDNA synthesis is warranted in order to draw to draw rigorous conclusions on Defa mRNA levels in extra-small intestinal tissues (e.g., colon) with low-level expression (that is, $10 \mathrm{e} 3 \mathrm{mRNA}$ copies or less per $10 \mathrm{ng}$ mRNA with external standards, or with relative levels of $\sim 0.001$ or less compared to Actb expression).

In summary, the methodological approach reported here utilizes external standards and generates assessment of absolute concentrations of specific $\alpha$-defensin mRNA in experimental tissues. The approach overcomes the technological challenge of highly similar $\alpha$-defensin paralogs in C57BL6 mice and offers a rigorous means to compare Paneth cell $\alpha$-defensin mRNA expression in investigations in separate laboratories. In some circumstances, simply using the oligonucleotide primers and conditions presented in Table 1, together with being mindful of the anatomic and developmental expression patterns elucidated here, should provide investigators with higher confidence of rigor in data interpretation.

\section{Methods}

Mice. The Institutional Animal Care and Use Committee at the University of California, Davis, approved all procedures involving live animals and methods of euthanasia; experiments were performed in accordance with these approved procedures. The genetic background of all mice used in these experiments was C57BL/6. Dr. Andreas Bäumler and members of his laboratory generously provided small intestinal tissue (Figs 4 and 5) from germ-free (Taconic Biosciences, Germantown, NY), streptomycin-treated ${ }^{72}$ and MyD88 $8^{-1-}$ mice $^{73}$. Tissues for experiments of Figs 3 and 6 were from a specific-pathogen free mouse colony at UC Davis.

RNA isolation and reverse transcription. The general procedures for RNA isolation and synthesis of cDNA were previously described by our group ${ }^{41}$. Briefly, intestinal tissue samples were dissected immediately after mice were euthanized and placed in RNAlater $(\sim 1: 10 \mathrm{w} / \mathrm{v}$, Sigma Aldrich, St. Louis, MO). The RNAlater specimen tubes were incubated with gentle rocking overnight at $4{ }^{\circ} \mathrm{C}$, and then stored at $-20^{\circ} \mathrm{C}$. For processing, the RNAlater solution was decanted and the tissue was homogenized in guanidine thiocyanate buffer ${ }^{41,74}$. Total RNA was isolated using cesium chloride gradient ultracentrifugation ${ }^{41}$, and then quantified using ultraviolet absorption spectrometry at $260 \mathrm{~nm}$. For cDNA synthesis, 1 to $5 \mu \mathrm{g}$ of total RNA was reverse transcribed using Superscript II reverse transcriptase (Invitrogen, Carlsbad, CA) using an oligo- $(\mathrm{dT})_{12-18} \operatorname{primer}^{41}$. The single-stranded cDNA product was purified using Qiagen PCR purification kit (Qiagen, Valencia, CA), and diluted to $10 \mathrm{ng} / \mu \mathrm{l}$ based on the input concentration of total RNA. Previous control experiments by our group demonstrated that when RNA from a single specimen was used to independently synthesize, isolate and purify the cDNA, the reaction-to-reaction variability was $\leq 15 \%{ }^{41}$. Other reproducibility assessments of this approach were previously reported ${ }^{41}$.

In experiments to interrogate the possible role of contaminating DNA as confounding factor in qRT-PCR assays, total RNA ( $1 \mu \mathrm{g})$ was treated with DNase I (1 units, Thermo Fisher Scientific, Waltham, Massachusetts) for 30 minutes at $37^{\circ} \mathrm{C}$ and then the enzyme was inactivated according to the manufacturer's suggestions. The resulting RNA sample was used as a template for reverse transcription as described above.

For analysis of expression during development, intestinal tissues were isolated at postnatal day (PD) 5, 10, 20, and 50. Immediately after euthanasia, the intestine was flushed with PBS to clear luminal contents. For PD5, PD10 and PD20, the small intestine was then sectioned equally into proximal and distal portions and then processed as above. For PD 50 (adult) mice, $10 \mathrm{~cm}$ of the most proximal small intestine, $10 \mathrm{~cm}$ of the most distal small intestine, the proximal half of the large intestine and the distal half of the large intestine were dissected, and then processed as above.

Generation of Defa standard curves. Primers that target C57BL/6 Defa mRNA (Supplementary Table S3) were designed to amplify the collection of Defa cDNAs from small intestinal mRNA in four reactions, each yielding mixed PCR products. The PCR reaction conditions were $95^{\circ} \mathrm{C}$ for $5 \mathrm{~min}$, followed by 35 cycles of $95^{\circ} \mathrm{C}$, $30 \mathrm{sec}$, annealing for $30 \mathrm{sec}$ at $58^{\circ} \mathrm{C}$ and extension at $72^{\circ} \mathrm{C}$ for $90 \mathrm{sec}$. Final extension time was $7 \mathrm{~min}$ at $72^{\circ} \mathrm{C}$. The resulting PCR product pools were then ligated into a pBluescript II s/k plasmid vector (Agilent Technologies, Stratagene Products Division, La Jolla, CA $)^{75,76}$ and transformed into E. coli cells. Multiple clones from each PCR reaction were isolated and the plasmid DNA from the clones was purified and sequenced in both directions to determine the identity of each clone. This yielded eight targeted Defa cDNAs for use as external standards (Defa3, Defa5, Defa20, Defa21, Defa22, Defa23, Defa24, and Defa26). The Defa specific plasmids were then quantified by UV spectroscopy, the molar concentration of each Defa cDNA insert determined, and then 10-fold serial dilutions were performed using yeast RNA $(0.2 \mu \mathrm{l} / \mu \mathrm{l})$ as a carrier nucleic acid in the diluent. These dilutions provided templates used in real-time PCR with gene-specific PCR primers (Table 1) in order to generate standard curves for each reaction ${ }^{41}$. Using Avogadro's constant, the number of Defa target sequence copies were calculated for each of the standard curve solutions. The Defa gene-specific PCR primer pairs for the eight target cDNAs were designed, based initially on those reported by Menendez et al. ${ }^{39}$, and then substantially modified to improve target specificity (Table 1).

Quantitative real-time RT-PCR of Paneth cell products. Gene-specific oligonucleotide primers (Tables 1 and 2) were synthesized by Invitrogen Life Technologies (Carlsbad, CA). Design of these primers used MacVector software (MacVector, Cary, NC), except for those in Table 1 designated as from Menendez, et al..$^{39}$. Real-time PCR was performed using single-stranded cDNA from experimental tissues or gene-specific plasmids (from the standard curve described above) as templates, using the specific oligonucleotide primer pairs and LightCycler FastStart DNA Master SYBR Green 1 reagents (with $\left[\mathrm{MgCl}_{2}\right]=1 \mathrm{mM}$, final) in a thermocycler equipped with a fluorescence detection monitor (Light Cycler, Roche Diagnostics, Mannheim, Germany) as described ${ }^{41}$. Accumulation of fluorescence signal from intercalation of the SYBR green probe into the PCR 
products was thereby monitored cycle-by-cycle. Absolute quantification of specific mRNA from tissue was determined by extrapolation of the detection threshold (crossing point) to the crossing point for gene-specific external plasmid standard analyzed within each $\operatorname{run}^{41}$. A negative control reaction that omitted template cDNA was included with each set of reactions to check for possible cross-contamination. To confirm PCR amplification of the intended product, melting temperature profile curves of every PCR reaction was determined at the end of each reaction as described ${ }^{41}$.

Statistical analysis. Error bars represent standard error of the mean. For non-parametric statistical comparisons of data between two groups, a Mann Whitney test was performed using Prism 8 (GraphPad Software, Inc). Stata version 12.1 (Statacorp, College Station TX) was used to generate principal component analyses; scree plots of Eigen values were generated to determine the number of components.

\section{References}

1. Peterson, L. W. \& Artis, D. Intestinal epithelial cells: regulators of barrier function and immune homeostasis. Nat Rev Immunol 14, 141-153 (2014).

2. Ley, R. E., Lozupone, C. A., Hamady, M., Knight, R. \& Gordon, J. I. Worlds within worlds: evolution of the vertebrate gut microbiota. Nat Rev Microbiol 6, 776-788 (2008)

3. Hooper, L. V., Littman, D. R. \& Macpherson, A. J. Interactions between the microbiota and the immune system. Science 336, $1268-1273$ (2012).

4. Kamada, N., Seo, S. U., Chen, G. Y. \& Nunez, G. Role of the gut microbiota in immunity and inflammatory disease. Nat Rev Immunol 13, 321-335 (2013).

5. Sommer, F. \& Backhed, F. The gut microbiota-masters of host development and physiology. Nat Rev Microbiol 11, 227-238 (2013).

6. Chu, H. \& Mazmanian, S. K. Innate immune recognition of the microbiota promotes host-microbial symbiosis. Nat Immunol 14, 668-675 (2013)

7. Subramanian, S. et al. Cultivating Healthy Growth and Nutrition through the Gut Microbiota. Cell 161, 36-48 (2015).

8. Schroeder, B. O. \& Backhed, F. Signals from the gut microbiota to distant organs in physiology and disease. Nat Med 22, 1079-1089 (2016).

9. Pickard, J. M., Zeng, M. Y., Caruso, R. \& Nunez, G. Gut microbiota: Role in pathogen colonization, immune responses, and inflammatory disease. Immunol Rev 279, 70-89 (2017).

10. Packey, C. D. \& Sartor, R. B. Commensal bacteria, traditional and opportunistic pathogens, dysbiosis and bacterial killing in inflammatory bowel diseases. Current Opinion in Infectious Diseases 22, 292-301 (2009).

11. Sekirov, I. \& Finlay, B. B. The role of the intestinal microbiota in enteric infection. J Physiol 587, 4159-4167 (2009).

12. Plottel, C. S. \& Blaser, M. J. Microbiome and Malignancy. Cell Host \& Microbe 10, 324-335 (2011).

13. Mathis, D. \& Benoist, C. The influence of the microbiota on type-1 diabetes: on the threshold of a leap forward in our understanding. Immunological Reviews 245, 239-249 (2012).

14. Pflughoeft, K. J. \& Versalovic, J. Human microbiome in health and disease. Annu Rev Pathol 7, 99-122 (2012).

15. Schwabe, R. F. \& Jobin, C. The microbiome and cancer. Nat Rev Cancer 13, 800-812 (2013).

16. Goodrich, J. K. et al. Human genetics shape the gut microbiome. Cell 159, 789-799 (2014).

17. Shreiner, A. B., Kao, J. Y. \& Young, V. B. The gut microbiome in health and in disease. Curr Opin Gastroenterol 31, 69-75 (2015)

18. Sonnenburg, J. L. \& Backhed, F. Diet-microbiota interactions as moderators of human metabolism. Nature 535, 56-64 (2016).

19. Sheehan, D. \& Shanahan, F. The Gut Microbiota in Inflammatory Bowel Disease. Gastroenterol Clin North Am 46, 143-154 (2017).

20. Kim, D., Zeng, M. Y. \& Nunez, G. The interplay between host immune cells and gut microbiota in chronic inflammatory diseases. Experimental \& molecular medicine 49, e339 (2017).

21. Jonsson, A. L. \& Backhed, F. Role of gut microbiota in atherosclerosis. Nature reviews. Cardiology 14, 79-87 (2017).

22. Griffiths, J. A. \& Mazmanian, S. K. Emerging evidence linking the gut microbiome to neurologic disorders. Genome Med 10, 98 (2018).

23. Robertson, R. C., Manges, A. R., Finlay, B. B. \& Prendergast, A. J. The Human Microbiome and Child Growth - First 1000 Days and Beyond. Trends Microbiol 27, 131-147 (2019).

24. Salzman, N. H. et al. Enteric defensins are essential regulators of intestinal microbial ecology. Nat Immunol 11, 76-83 (2010).

25. Bevins, C. L. \& Salzman, N. H. The potter's wheel: the host's role in sculpting its microbiota. Cell Mol Life Sci 68, 3675-3685 (2011).

26. Schluter, J. \& Foster, K. R. The evolution of mutualism in gut microbiota via host epithelial selection. PLoS Biol 10, e1001424 (2012).

27. Litvak, Y., Byndloss, M. X. \& Baumler, A. J. Colonocyte metabolism shapes the gut microbiota. Science 362, eaat9076 (2018).

28. Ouellette, A. J. Paneth cell alpha-defensins in enteric innate immunity. Cell Mol Life Sci 68, 2215-2229 (2011).

29. Bevins, C. L. \& Salzman, N. H. Paneth cells, antimicrobial peptides and maintenance of intestinal homeostasis. Nat Rev Microbiol 9 , 356-368 (2011)

30. Clevers, H. C. \& Bevins, C. L. Paneth cells: maestros of the small intestinal crypts. Annu Rev Physiol 75, 289-311 (2013).

31. Porter, E. M., Bevins, C. L., Ghosh, D. \& Ganz, T. The multifaceted Paneth cell. Cell Mol Life Sci 59, 156-170 (2002).

32. Bevins, C. L. Innate immune functions of alpha-defensins in the small intestine. Dig Dis 31, 299-304 (2013).

33. Wilson, C. L. et al. Regulation of intestinal alpha-defensin activation by the metalloproteinase matrilysin in innate host defense. Science 286, 113-117 (1999).

34. Salzman, N. H., Ghosh, D., Huttner, K. M., Paterson, Y. \& Bevins, C. L. Protection against enteric salmonellosis in transgenic mice expressing a human intestinal defensin. Nature 422, 522-526 (2003).

35. Vaishnava, S., Behrendt, C. L., Ismail, A. S., Eckmann, L. \& Hooper, L. V. Paneth cells directly sense gut commensals and maintain homeostasis at the intestinal host-microbial interface. Proc Natl Acad Sci USA 105, 20858-20863 (2008).

36. Malakoff, D. The rise of the mouse, biomedicine's model mammal. Science 288, 248-253 (2000).

37. Amid, C. et al. Manual annotation and analysis of the defensin gene cluster in the C57BL/6J mouse reference genome. BMC Genomics 10, e606 (2009)

38. Shanahan, M. T., Tanabe, H. \& Ouellette, A. J. Strain-Specific Polymorphisms in Paneth Cell \{alpha\}-Defensins of C57BL/6 Mice and Evidence of Vestigial Myeloid \{alpha\}-Defensin Pseudogenes. Infect Immun 79, 459-473 (2011).

39. Menendez, A. et al. Bacterial stimulation of the TLR-MyD88 pathway modulates the homeostatic expression of ileal Paneth cell alpha-defensins. J Innate Immun 5, 39-49 (2013).

40. Gulati, A. S. et al. Mouse background strain profoundly influences Paneth cell function and intestinal microbial composition. PLoS ONE 7, e32403 (2012).

41. Wehkamp, J. et al. Paneth cell antimicrobial peptides: Topographical distribution and quantification in human gastrointestinal tissues. FEBS Letters 580, 5344-5350 (2006).

42. Bustin, S. A. Absolute quantification of mRNA using real-time reverse transcription polymerase chain reaction assays. $J$ Mol Endocrinol 25, 169-193 (2000).

43. Radonic, A. et al. Guideline to reference gene selection for quantitative real-time PCR. Biochem Biophys Res Commun 313, 856-862 (2004). 
44. Darmoul, D. \& Ouellette, A. J. Positional specificity of defensin gene expression reveals Paneth cell heterogeneity in mouse small intestine. Am J Physiol 271, G68-74 (1996).

45. Inoue, R. et al. Postnatal changes in the expression of genes for cryptdins 1-6 and the role of luminal bacteria in cryptdin gene expression in mouse small intestine. FEMS Immunol Med Microbiol 52, 407-416 (2008).

46. Karlsson, J. et al. Regional variations in Paneth cell antimicrobial peptide expression along the mouse intestinal tract. BMC Immunol 9, e37 (2008)

47. Hornef, M. W., Putsep, K., Karlsson, J., Refai, E. \& Andersson, M. Increased diversity of intestinal antimicrobial peptides by covalent dimer formation. Nat Immunol 5, 836-843 (2004).

48. Saeki, T. et al. Characterization, cDNA cloning, and functional expression of mouse ileal sodium-dependent bile acid transporter. $J$ Biochem 125, 846-851 (1999).

49. Boman, H. G. Innate immunity and the normal microflora. Immunol. Rev. 173, 5-16 (2000).

50. Kamada, N., Chen, G. Y., Inohara, N. \& Nunez, G. Control of pathogens and pathobionts by the gut microbiota. Nat Immunol 14, 685-690 (2013)

51. Round, J. L. \& Mazmanian, S. K. The gut microbiota shapes intestinal immune responses during health and disease. Nat Rev Immunol 9, 313-323 (2009).

52. Smith, K., McCoy, K. D. \& Macpherson, A. J. Use of axenic animals in studying the adaptation of mammals to their commensal intestinal microbiota. Semin Immunol 19, 59-69 (2007).

53. Cash, H. L., Whitham, C. V., Behrendt, C. L. \& Hooper, L. V. Symbiotic bacteria direct expression of an intestinal bactericidal lectin. Science 313, 1126-1130 (2006).

54. Ouellette, A. J. et al. Developmental regulation of cryptdin, a corticostatin/defensin precursor mRNA in mouse small intestinal crypt epithelium. J. Cell. Biol. 108, 1687-1695 (1989).

55. Larsson, E. et al. Analysis of gut microbial regulation of host gene expression along the length of the gut and regulation of gut microbial ecology through MyD88. Gut 61, 1124-1131 (2012).

56. Natividad, J. M. et al. Differential induction of antimicrobial REGIII by the intestinal microbiota and Bifidobacterium breve NCC2950. Appl Environ Microbiol 79, 7745-7754 (2013).

57. Bohnhoff, M., Drake, B. L. \& Miller, C. P. Effect of streptomycin on susceptibility of intestinal tract to experimental Salmonella infection. Proc Soc Exp Biol Med 86, 132-137 (1954).

58. Hapfelmeier, S. \& Hardt, W. D. A mouse model for S-typhimurium-induced enterocolitis. Trends in Microbiology 13, 497-503 (2005).

59. Olsan, E. E. et al. Colonization resistance: The deconvolution of a complex trait. J Biol Chem 292, 8577-8581 (2017).

60. Spees, A. M., Lopez, C. A., Kingsbury, D. D., Winter, S. E. \& Baumler, A. J. Colonization resistance: battle of the bugs or Menage a Trois with the host? PLoS Pathog 9, e1003730 (2013).

61. Stockinger, S. et al. TRIF signaling drives homeostatic intestinal epithelial antimicrobial peptide expression. J Immunol 193, 4223-4234 (2014).

62. Brandl, K., Plitas, G., Schnabl, B., DeMatteo, R. P. \& Pamer, E. G. MyD88-mediated signals induce the bactericidal lectin RegIII gamma and protect mice against intestinal Listeria monocytogenes infection. J Exp Med 204, 1891-1900 (2007).

63. Bry, L. et al. Paneth cell differentiation in the developing intestine of normal and transgenic mice. Proc Natl Acad Sci USA 91, 10335-10339 (1994).

64. Darmoul, D., Brown, D., Selsted, M. E. \& Ouellette, A. J. Cryptdin gene expression in developing mouse small intestine. Am J Physiol 272, G197-206 (1997).

65. Ouellette, A. J. Peptide localization and gene structure of cryptdin 4, a differentially expressed mouse paneth cell alpha-defensin. Infect Immun 67, 6643-6651 (1999).

66. Ouellette, A. J. et al. Mouse Paneth cell defensins: primary structures and antibacterial activities of numerous cryptdin isoforms. Infect. Immun. 62, 5040-5047 (1994).

67. Haber, A. L. et al. A single-cell survey of the small intestinal epithelium. Nature 551, 333-339 (2017).

68. Yu, T. et al. Association of Bactericidal Dysfunction of Paneth Cells in Streptozocin-Induced Diabetic Mice with Insulin Deficiency. Med Sci Monit 22, 3062-3072 (2016).

69. Schoenborn, A. A. et al. The enteric microbiota regulates jejunal Paneth cell number and function without impacting intestinal stem cells. Gut Microbes 10, 45-58 (2019).

70. Stanford, A. H. et al. A direct comparison of mouse and human intestinal development using epithelial gene expression patterns. Pediatr Res, https://doi.org/10.1038/s41390-41019-40472-y. [Epub ahead of print] (2019).

71. Kaiser, P., Diard, M., Stecher, B. \& Hardt, W. D. The streptomycin mouse model for Salmonella diarrhea: functional analysis of the microbiota, the pathogen's virulence factors, and the host's mucosal immune response. Immunol Rev 245, 56-83 (2012).

72. Spees, A. M. et al. Streptomycin-Induced Inflammation Enhances Escherichia coli Gut Colonization Through Nitrate Respiration. MBio 4, e00430-00413 (2013).

73. Keestra, A. M. et al. Early MyD88-dependent induction of interleukin-17A expression during Salmonella colitis. Infect Immun 79, 3131-3140 (2011).

74. Chirgwin, J. M., Pryzybyla, A. E., MacDonald, R. J. \& Rutter, W. J. Isolation of biologically active ribonucleic acid from sources enriched in ribonuclease. Biochem. 18, 5294-5299 (1979).

75. Jones, D. E. \& Bevins, C. L. Paneth cells of the human small intestine express an antimicrobial peptide gene. J Biol Chem 267, 23216-23225 (1992).

76. Mallow, E. B. et al. Human enteric defensins. Gene structure and developmental expression. J Biol Chem 271, 4038-4045 (1996).

77. Wehkamp, J. et al. The Paneth Cell \{alpha\}-Defensin Deficiency of Ileal Crohn's Disease Is Linked to Wnt/Tcf-4. The Journal of Immunology 179, 3109-3118 (2007).

\section{Acknowledgements}

This work was supported by The National Institutes of Health (R21AI099519 (C.L.B.), R37AI32738 (C.L.B.), and the Mucosal Immunology Study Team U01AI125596 (C.L.B. and B.L.)).

\section{Author Contributions}

P.A.C. and C.L.B. conceived the idea of this study, designed the experiments, interpreted the data and wrote the initial draft of the paper. P.A.C. oversaw the details of all experiments. E.B.N. contributed ideas, performed experiments, interpreted and graphed data, and helped write the paper. Under the supervision of P.A.C., D.T.O. cloned the eight cDNA standards, and M.T.N.T. and S.M.G. performed some qRT-PCR reactions. M.A.U. performed analysis of the data and contributed to writing the paper. B.L. and C.L.B. were responsible for supervisory oversight and obtaining grant support. All authors concur with the interpretation of data and with the contents of the final version of the manuscript. 


\section{Additional Information}

Supplementary information accompanies this paper at https://doi.org/10.1038/s41598-019-49471-9.

Competing Interests: The authors declare no competing interests.

Publisher's note: Springer Nature remains neutral with regard to jurisdictional claims in published maps and institutional affiliations.

(c) (i) Open Access This article is licensed under a Creative Commons Attribution 4.0 International License, which permits use, sharing, adaptation, distribution and reproduction in any medium or format, as long as you give appropriate credit to the original author(s) and the source, provide a link to the Creative Commons license, and indicate if changes were made. The images or other third party material in this article are included in the article's Creative Commons license, unless indicated otherwise in a credit line to the material. If material is not included in the article's Creative Commons license and your intended use is not permitted by statutory regulation or exceeds the permitted use, you will need to obtain permission directly from the copyright holder. To view a copy of this license, visit http://creativecommons.org/licenses/by/4.0/.

(C) The Author(s) 2019 